\title{
Investigating Player Experience in Virtual Reality Games via Remote Experimentation
}

\author{
IVAN IP and PENNY SWEETSER, Australian National University, Australia
}

This research explores player experience of virtual reality (VR) games through two stages of study. In both stages, we employed the Player Experience Inventory (PXI), a validated tool designed to evaluate player experience. In Stage 1, player experience of VR games was investigated via an online survey with 100 participants. We found that Audio-Visual Appeal, Immersion, and Ease of Control contributed most to player experience in VR games. We found no relationship between player experience and age, time spent playing, VR experience, or VR headset. Stage 2 used remote experimentation to compare VR and non-VR games with 10 participants. We found that differences in player experience can be explained by the Immersion, Progress Feedback, and Curiosity constructs of the PXI.

CCS Concepts: • Human-centered computing $\rightarrow$ User studies; Virtual reality; • Software and its engineering $\rightarrow$ Interactive games.

Additional Key Words and Phrases: virtual reality; video games; enjoyment; player experience

\section{ACM Reference Format:}

Ivan Ip and Penny Sweetser. 2021. Investigating Player Experience in Virtual Reality Games via Remote Experimentation. In Extended Abstracts of the 2021 Annual Symposium on Computer-Human Interaction in Play (CHI PLAY '21), October 18-21, 2021, Virtual Event, Austria. ACM, New York, NY, USA, 9 pages. https://doi.org/10.1145/3450337.3483462

\section{INTRODUCTION}

The emergence of virtual reality (VR) from the late 20th century has revolutionised the perception of how technology can be used in various domains, including applications for entertainment and education. VR is a computer-generated experience that allows users to be immersed in and interact with the simulated environment, presenting many opportunities for application. For example, VR has been implemented for a variety of training purposes [3], including medical, military, and flight simulation. In 2020, the VR industry was valued at $\$ 15.8$ billion USD and projected to reach revenue of $\$ 62.1$ billion USD by 2027 [7]. Video games is the largest and most rapidly expanding entertainment industry, with the global market growing 9.3 per cent in 2020 to US $\$ 159.3$ billion and projected to exceed $\$ 200$ billion by 2023 [11]. Given the rapid growth in both games and VR, many companies have invested in developing optimised VR game systems, including industry leaders such as Google, Microsoft, Sony, and Facebook [7].

Despite the rapid growth of games and VR, there is limited research on player experience of VR games that involves comparing VR and non-VR experiences [9]. Exploration into player experience in VR games is important, as insights can lead to improvements in game design and usability, as well as potential improvements for the many non-game VR applications that are emerging. This research project aims to investigate the effect of VR on player experience in video games, with a particular focus on comparing VR and non-VR game experiences across a variety of game experiences. As this is a relatively new area of study, this research project aims to investigate the effect of VR on player experience, using the Player Experience Inventory (PXI) [1]. The research was divided into two studies: Stage 1 Online

Permission to make digital or hard copies of part or all of this work for personal or classroom use is granted without fee provided that copies are not made or distributed for profit or commercial advantage and that copies bear this notice and the full citation on the first page. Copyrights for third-party components of this work must be honored. For all other uses, contact the owner/author(s).

(c) 2021 Copyright held by the owner/author(s).

Manuscript submitted to ACM 
Surveying and Stage 2 Remote Experimentation. Stage 1 employs an online survey of 100 participants to investigate the relationship between VR player experience, measured by the PXI, and the demographic variables of age, weekly time spent playing games, and experience with VR games, as well as the recency of the VR headset used. Stage 2 involves remote experimentation with 10 participants to compare player experiences between VR and non-VR games through the breakdown of the PXI constructs. Due to COVID-19, our approach was entirely online, including remote experimentation in Stage 2.

Our online surveys and remote experimentation approach provided interesting initial results on the relevance of certain constructs of the PXI for VR games, as well as the relationship between the player experience and general predictor variables. We found that Audio-Visual Appeal, Immersion, and Ease of Control contributed most to player experience in VR games in Stage 1 and that differences in player experience can be explained by the Immersion, Progress Feedback, and Curiosity constructs of the PXI in Stage 2. This research contributes to the understanding of VR player experience and provides a foundation for future research into VR player experience and analysis of the PXI constructs.

\section{RELATED WORK}

There has been limited previous research into general player experience in VR games, particularly in terms of direct comparisons to non-VR games [9]. Most previous research has focused on a single game experience [4, 8]. Other limitations include a small number of participants [4] and analysis of passive data (game reviews) [9]. In previous research investigating the effect of VR on game user satisfaction [8], participants played the game Defence Grid 2 using both a VR system and a standard PC setup. They were then tasked with completing the Game User Experience Satisfaction Scale (GUESS), a survey designed to measure user satisfaction. The study found that playing the VR version of the game generally resulted in a more satisfying experience when compared to the same game played on PC [8]. However, the study was limited to a single game, Defence Grid 2, which belongs to the Tower Defence genre. In order to extend previous research into VR versus non-VR experience, our research aims to examine a variety of games across different genres.

A similar study [4] compared the game Smash Hit on tablet and VR using self-report questionnaires. It was found that participants perceived the VR experience to be more appealing, but less usable, than the tablet version [4]. Furthermore, the participants reported a higher level of anxiety after playing in VR than on tablet, which might be related to their lack of familiarity with playing in VR [4]. A relatively small sample size of 10 participants was involved, in addition to focusing on a single game. Moreover, the demographic of the participants were young adults who played video games casually and had little knowledge or experience of VR technology. Taking these limitations into consideration, our research project aims to draw on a larger participant base and include players with more VR experience.

Another recent study investigated differences in player experience between VR and non-VR versions of two games, SuperHot and Skyrim [9]. A qualitative analysis of 40 professional game reviews was conducted, using GameFlow [10] as a theoretical framework. The analysis revealed differences in relation to the GameFlow elements of Immersion, Feedback, Player Skills, and Control, in addition to proposing a new element of Comfort. Although more than one game and more experienced players (game reviewers) were investigated, the method involved passive data use rather than experimentation and direct data collection from participants.

\section{PLAYER EXPERIENCE INVENTORY (PXI)}

The PXI was designed to allow for an understanding of how the interpretation of game design choices by players ultimately affects their psychological experiences [1]. The PXI measures both functional and psychosocial consequences 
of game design choices. Functional consequences are described as "the immediate, tangible consequences" and psychosocial consequences as "emotional experiences". During development and validation, the PXI underwent 7 studies involving 529 participants and 64 game user research experts. The resulting PXI model is comprised of 30 items, each scored on a 7-point Likert scale, to measure the functional and psychosocial consequences:

- Functional Consequences: Ease of Control (3 items), Challenge (3 items), Progress Feedback (3 items), Goals and Rules (3 items), Audio-visual Appeal (3 items)

- Psychosocial Consequences: Meaning (3 items), Immersion (3 items), Mastery (3 items), Curiosity (3 items), Autonomy (3 items)

The PXI was chosen to measure player experience for this research, as it is a validated measure of both the functional and psychosocial consequence levels of player experience. Given that the PXI was developed and validated using traditional video games, applying it to VR games is also an interesting test of its applicability to new technologies. GUESS [5] was also considered, as it is employed in previous VR games research [8]. However, it focuses on user satisfaction, rather than player experience, and is more suited to comparing games within the same genre [5].

\section{EXPERIMENTAL DESIGN \& METHOD}

We had originally planned to conduct in-person experiments, involving participants playing the same game (from a selection) on both VR and standard game systems. However, due to the global outbreak of COVID-19, we revised our approach to work remotely. Our research was conducted in two stages: Stage 1 - Online Surveying; and Stage 2 Remote Experimentation. Given the remote nature of both stages, participants were required to have access to a VR game system to be eligible. Additionally, Stage 2 participants were required to have access to a standard game system.

Both stages employed the PXI to measure player experience. Stage 1 aimed to gain a general understanding of player experience in VR games by investigating the relationships between player experience (PXI score) and selected variables, including VR games experience, VR headset, and player age. Stage 2 compared player experience in VR and standard games. The two stages were designed to investigate the following questions and hypotheses:

Stage 1: H1: that a more recent VR headset is associated with a higher PXI score. H2: that more experience with VR is associated with a higher PXI score. Q1: is there a relationship between PXI score and age? Q2: is there a relationship between PXI score and average game playing time per week? Q3: which PXI constructs have the most influence on player experience of VR games?

Stage 2: Q4: how does player experience differ between VR and non-VR games? Q5: how do the PXI constructs differ between VR and non-VR games?

\subsection{Stage 1: Online Surveying}

Stage 1 of the research involved recruiting participants to complete an online survey, including demographics, playing experience, selected game details, and the PXI. Participants were instructed to complete the survey based on a recent experience with playing a VR game. Given the specific set of requirements for study eligibility (such as having access to VR technology), online VR communities were approached to advertise the research. AV Forums [2] and Reddit [6] were identified as being the most active online communities relating to VR. As such, the study was advertised within these two communities. As Reddit is comprised of many specific 'subreddits', where discussions on particular topics take place, the study was presented to many VR system-based and general VR subreddits such as r/PSVR and r/virtualreality. 


\subsection{Stage 2: Remote Experimentation}

Stage 2 involved recruiting participants to take part in remote experimentation. Participants were asked to read the Participant Information Sheet and the Remote Experiment Instructions, which instructed them to fill out the Preliminary Study Form, play a VR game of their choosing for 30 minutes, followed by playing a comparable video game for 30 minutes. The participants were instructed to complete the PXI after playing each game and finally complete the Post-Experiment Survey. The selected video game was played on a PC or console such as Xbox, PlayStation, or similar. Participants were asked to choose one of the three levels of "game comparability" from the following list, ordered by priority:

- Priority 1 - Same Game: Video game to be the exact same game as the chosen VR game.

- Priority 2 - Same Series: Video game to be part of the same series as the chosen VR game.

- Priority 3 - Same Genre: Video game to share the same genre as the chosen VR game.

Of course, if participants followed Priority 1, playing VR and non VR versions of the same game, then their experiences would be more comparable. However, given that not all VR games have a non-VR version and that participants were relying on their own systems and resources, more flexibility was provided via the other selection criteria. Given this flexibility, participants were required to briefly describe the in-game tasks that were performed during their playtime of both the VR and non-VR games at the end of the study.

Preliminary study questions included participant demographics, game playing frequency, experience with VR games, nominated VR game and system to be used for the study, selected level of "game comparability", and nominated non-VR game and system to be used for the study. Post-Experiment questions included whether the participant experienced any latency issues throughout experiment, general comments on the comparison of their experiences of VR and non-VR games during experiment, any nausea or cybersickness experienced, what tasks were done in each game, and which experience they found more enjoyable.

\section{DATA COLLECTION \& ANALYSIS}

As both stages of the experiment were designed for remote participation, Google Forms was used as a platform to distribute the surveys to participants and gather data. The surveys for both stages remained available for 13 days in August 2020. During this time, a total of 101 responses were recorded for Stage 1 and 10 responses were recorded for Stage 2. Of the 101 responses received for Stage 1, one of the responses was not appropriate and did not relate to the study. As such, this case was treated as irrelevant data and subsequently removed from the sample. For Stage 2, 10 responses were received.

\subsection{Participants}

The remote nature of the experiment facilitated more diversity in participant background with differing experiences in VR games. A range of demographic data was seen in the country, age, and game playing experience of the participants in both stages. Of the 100 responses obtained for Stage 1, the most common participant country was the USA at 39\%, followed by Australia at $16 \%$. In total, there were 24 countries among the responses received. An average age of 27 was recorded, with a minimum age of 11 and a maximum of 59. The majority of participants (49\%) were young people below the age of 25 , many of who were students. The other half of responses were comprised of individuals in their $30 \mathrm{~s}, 40 \mathrm{~s}$, and 50s who play games using VR. On average, participants typically spent 14.5 hours per week playing games (all 
Table 1. Average Scores and Estimated Regression Coefficients for the individual PXI constructs related to Functional consequences (top) and Psychosocial consequences (bottom).

\begin{tabular}{lcc}
\hline PXI Construct & Average & Coeff. \\
\hline Ease of Control & 5.89 & 2.85 \\
Challenge & 5.66 & 2.78 \\
Progress Feedback & 5.46 & 2.69 \\
Goals \& Rules & 6.10 & 3.09 \\
Audio-Visual Appeal & 6.44 & 3.77 \\
\hline Meaning & 5.93 & 2.91 \\
Immersion & 6.10 & 3.08 \\
Mastery & 5.68 & 3.12 \\
Curiosity & 5.63 & 2.39 \\
Autonomy & 5.59 & 1.85 \\
\hline
\end{tabular}

types). Participants were asked how long ago they first used VR for playing games, as an indicator of their familiarity with VR games. Stage 1 participants had two years of VR game playing experience on average.

Of the 10 responses obtained for Stage 2, the most common participant country was the USA at 40\%, with Australia next at $20 \%$. There were 5 countries in total among the responses received. An average age of 29 was recorded for Stage 2 , with a minimum age of 16 and a maximum of 49 . The majority of participants (60\%) were above the age of 25 . On average, participants in Stage 2 typically spent 13 hours per week playing games (all types). Stage 2 participants had at least one year of VR game playing experience on average.

\subsection{Stage 1 Analysis}

For Stage 1, the PXI score was regressed against the demographic variables of age, average time spent playing games per week, and experience with VR games. Hypothesis testing was also performed alongside the regression to determine whether a linear relationship could be observed between the PXI score response variable and the corresponding demographic predictor variables. No significant relationships were found between the PXI score and the demographic predictor variables ( $p$-values for age $=0.287$, weekly hours $=0.619$, VR game experience $=0.973$ ). A segmented data study of the most popular game selected, Beat Saber with 25 responses, was also analysed to detect any within-game trends. The same regression modelling and hypothesis tests were performed, which reaffirmed the broader results that there appears to be no linear relationship between the PXI score response variable and the demographic predictor variables of age, weekly game playing hours, and experience with VR games. A box and whisker plot was constructed to observe if the recency of the VR headset used affected the player experience. There was no significant trend found.

5.2.1 PXI Construct Analysis. An analysis of the individual PXI constructs and their contribution towards the PXI score was performed. The scores of each construct were averaged for all responses in Stage 1 (see Table 1). The Goals \& Rules and Audio-Visual Appeal constructs recorded the highest average scores from the set of constructs forming the Functional consequences. For the Psychosocial consequences, the Immersion and Meaning constructs were identified as the two factors with the highest average scores. Analysing construct score averages only informs the prominent constructs at face value, not its sensitivity to the PXI score. In this context, the greater the construct sensitivity, the more positive the relationship between that particular construct and the PXI score. Consequently, the PXI scores were regressed against the individual constructs. By definition, the PXI is composed of these individual constructs. As such, 
Table 2. Comparison of VR and Non-VR PXI Construct averages for all responses (left) and "same game" group (right).

\begin{tabular}{l|ccc|ccc|} 
& \multicolumn{3}{|c|}{ All } & \multicolumn{3}{c|}{ Same } \\
\hline PXI Construct & VR & Non-VR & Diff. & VR & Non-VR & Diff. \\
\hline Ease of Control & 5.07 & 5.23 & -0.17 & 4.89 & 5.00 & -0.11 \\
Challenge & 4.73 & 4.13 & 0.60 & 4.78 & 4.61 & 0.17 \\
Progress F-back & 5.07 & 5.83 & -0.77 & 5.44 & 5.94 & -0.50 \\
Goals \& Rules & 6.10 & 6.10 & 0.00 & 5.94 & 6.00 & -0.06 \\
A-V Appeal & 5.77 & 5.90 & -0.13 & 6.44 & 6.50 & -0.06 \\
\hline Meaning & 5.80 & 5.27 & 0.53 & 6.06 & 5.83 & 0.22 \\
Immersion & 6.37 & 5.23 & 1.13 & 6.78 & 5.44 & 1.33 \\
Mastery & 6.23 & 5.50 & 0.73 & 6.22 & 5.78 & 0.44 \\
Curiosity & 5.50 & 4.77 & 0.73 & 5.67 & 5.06 & 0.61 \\
Autonomy & 5.37 & 4.93 & 0.43 & 5.78 & 5.33 & 0.44 \\
\hline
\end{tabular}

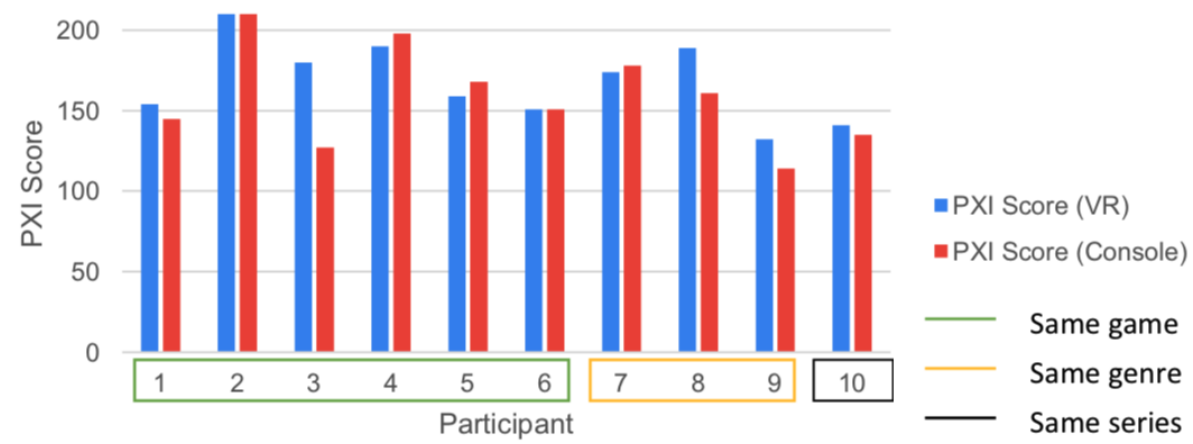

Fig. 1. PXI Score comparison between VR and non-VR Player Experiences.

Multiple Linear Regression would be redundant and provide little information on the sensitivity of each construct. Hence, to observe this sensitivity to PXI score, a Simple Linear Regression model was fit for each construct. The sensitivity analysis coefficients are also shown in Table 1 . The most sensitive constructs towards the PXI score in order are: Audio-Visual Appeal, Mastery, Goals \& Rules, Immersion, Meaning, and Ease of Control. These correspond to the top three most sensitive constructs for each of the Functional and Psychosocial consequences.

\subsection{Stage 2 Analysis}

The analysis of Stage 2 responses focused more on the breakdown of the PXI constructs with respect to the comparison of the VR and non-VR game playing experiences among the participants. Figure 1 illustrates the PXI score comparison between the VR and non-VR game playing experiences, showing varying levels of responses between the three groups. For the "same game" group, there is an even split between the experience preferences of VR, non-VR, and indifferent, with two responses for each category. For the "same genre" category, the PXI score for the VR experience was larger than the non-VR counterpart for the most part. The single participant who played games of the same series recorded PXI scores favouring the VR experience.

At the end, the survey also asked participants to indicate which experience they preferred or whether they felt indifferent. $70 \%$ indicated that they preferred the VR experience, $30 \%$ were indifferent, and none preferred the non-VR 
experience. In contrast, the PXI results indicated that 50\% of the responses favoured the VR experience, $30 \%$ favoured non-VR, and 20\% were indifferent. This might suggest that the PXI is not measuring a certain feature of player experience, particularly in relation to VR games. In addition, no participants recorded experiencing any latency or cybersickness issues, which might partly explain the sample's $70 \%$ preference for the VR experience.

5.3.1 PXI Construct Analysis. To investigate which PXI constructs were more important in explaining the PXI score for VR games, we examined the individual PXI construct scores for all responses and the "same game" responses (see Table 2). For all responses, the Immersion construct provided the greatest positive difference, meaning that the Immersion scores were on average much higher in the VR experience than the non-VR experience. This is to be expected given that VR technology interacts with the additional senses of depth perception and motion, compared to traditional games. This difference corresponds to just over one entire level on the Likert rating. Other notable constructs with absolute differences larger than 0.5 included Challenge and Progress Feedback from the Functional consequences and Meaning, Mastery, and Curiosity from the Psychosocial consequences. Although the Audio-Visual Appeal construct was identified as the most sensitive to the PXI score in Stage 1 for VR experiences, it is slightly favoured towards the non-VR experience for Stage 2.

For the "same game" group, the constructs with absolute differences greater than 0.5 were Progress Feedback from the Functional consequences and Immersion and Curiosity from the Psychosocial consequences. Although Immersion was shown to have the greatest difference, in line with the full sample, the Challenge, Meaning, and Mastery constructs all recorded difference averages below 0.5. As such, from this "same game" group, it appears that from using the PXI to evaluate player experience, the differences can be explained by the Immersion, Progress Feedback, and Curiosity constructs. The Functional consequences were also generally higher for VR, whereas the Psychosocial consequences were higher for non-VR.

\section{DISCUSSION}

Limited research has been published surrounding players' experience with VR games compared to non-VR games. This research project aimed to investigate the effect of VR on player experience, using the Player Experience Inventory (PXI). The research was divided into two studies: Stage 1 Online Surveying and Stage 2 Remote Experimentation. Stage 1 was designed to investigate the relationship between VR player experience, measured by the PXI, and participant age, weekly time spent playing games, experience with VR games, and recency of the VR headset used. The PXI constructs most influential to the sensitivity of the PXI score for VR games were also explored. Stage 2 focused on comparing the playing experiences between VR and non-VR through the breakdown of the PXI constructs.

Our analyses on the Stage 1 responses did not find any significant linear relationships between the PXI score and the predictor variables of age, weekly game playing hours, and experience with VR games. This was supported by a separate segmented data study on the game "Beat Saber" using the same analysis technique, to minimise the variance in experiences from different games. The answers to our proposed research questions Q1 and Q2 are therefore that no relationships were found between PXI score and age or game playing time per week.

Our hypotheses $\mathrm{H} 1$ and $\mathrm{H} 2$ were also not supported, as we found no relationships between PXI score and VR headset year or VR experience. Given the youth of the VR games industry and how different the controls and interface are to conventional games, it was hypothesised that the more experience an individual has with VR games, the more enjoyable they will find their VR game playing experience, as they would be more familiar with the controls and tolerate the potential cybersickness side-effects more easily. Similarly, we hypothesised that a more recent VR headset would 
support a better experience with fewer side effects. From the results of the regression analysis, there does not appear to be a linear relationship between player experience and experience with VR games or VR headset.

In order to answer our research question Q3, the PXI constructs were analysed. We found that the most sensitive constructs to the PXI score were Audio-Visual Appeal, Mastery, Goals \& Rules, Immersion, Meaning, and Ease of Control. Originally, it was expected that the Ease of Control, Audio-Visual Appeal, and Immersion constructs would provide the greatest influence towards evaluating the player experience of VR games. It is interesting to see that the Goals \& Rules construct is scored relatively highly as this particular factor was not considered to be related to VR technology but rather the game design.

Analysis on the Stage 2 responses suggested that the differences in PXI scores between VR and non-VR can be explained by the Immersion, Progress Feedback, and Curiosity constructs. Audio-Visual Appeal was scored relatively the same when comparing the same game on VR and non-VR. However, the contradictory results when comparing PXI scores to participant preferred experience suggest the PXI might not be capturing a certain element of player experience. This could also reflect limitations in our participant sample. We also observed that the Functional consequences favoured VR, whereas the Psychosocial consequences favoured non-VR, which is worth exploring in future research.

\subsection{Limitations \& Future Work}

The findings from both stages of the study provided insight into understanding player experience of VR games, through the use of the PXI. However, due to the limitations imposed by the remote experimentation approach, further studies are needed to reaffirm or challenge the results from this research. A larger sample size and in-person experiments to investigate the Stage 2 results would be beneficial. By shifting the study from remote to in-person, more variables can be controlled. The same game could be used so that the PXI scores can be compared to experiences from the same gameplay. This also enables the possibility of forming study groups for tests on causation. With the remote experiment, we also did not have control over the devices or duration of play. We provided participants with clear instructions, but there was a strong element of trust that participants were following the instructions and reporting accurately. The remote study required participants to have access to VR equipment and consequently, the responses were limited to people who have had some sort of prior experience with VR. An in-person study approach would eliminate this sample bias and allow for the recruitment of participants who have had no exposure to VR, which would be beneficial in analysing the PXI constructs for VR games. Participants were also playing games that they already owned, rather than experiencing games for the first time. An in-person study would allow a more diverse group of participants to be recruited, including participants with varying levels of VR experience and exposure to games that participants have not played previously.

\section{CONCLUSIONS}

Despite the limitations of having a small sample size of 10 and the nature of the remote study that necessitated the selection criteria for the games, our results seem to align with previous research $[4,8]$. The VR experience preference followed previous findings that VR generally results in a better experience compared to non-VR games. However, as the PXI scores were not consistent with the surveyed preferences, further studies comparing VR and non-VR on the same game with larger sample sizes are needed. The remote experimentation approach provided interesting initial results on the relevance of certain constructs towards the PXI score for VR games, as well as the relationship between player experience and general predictor variables. This research has contributed to the understanding of VR player experience and we hope that further studies will facilitate improved player experiences for future VR games. 


\section{REFERENCES}

[1] Vero Vanden Abeele, Katta Spiel, Lennart Nacke, Daniel Johnson, and Kathrin Gerling. 2020. Development and validation of the player experience inventory: A scale to measure player experiences at the level of functional and psychosocial consequences. International fournal of Human-Computer Studies 135 (2020), 102370. https://doi.org/10.1016/j.ijhcs.2019.102370

[2] AV Forums. 2021. AV Forums. Retrieved Jan 7, 2021 from https://www.avforums.com/

[3] Alexandra D. Kaplan, Jessica Cruit, Mica Endsley, Suzanne M. Beers, Ben D. Sawyer, and P. A. Hancock. 2020. The Effects of Virtual Reality, Augmented Reality, and Mixed Reality as Training Enhancement Methods: A Meta-Analysis. Human Factors (2020). https://doi.org/10.1177/0018720820904229 PMID: 32091937.

[4] Federica Pallavicini, Ambra Ferrari, Andrea Zini, Giacomo Garcea, Andrea Zanacchi, Gabriele Barone, and Fabrizia Mantovani. 2018. What Distinguishes a Traditional Gaming Experience from One in Virtual Reality? An Exploratory Study. In Advances in Human Factors in Wearable Technologies and Game Design, Tareq Ahram and Christianne Falcão (Eds.). Springer International Publishing, Cham, $225-231$.

[5] Mikki H. Phan, Joseph R. Keebler, and Barbara S. Chaparro. 2016. The Development and Validation of the Game User Experience Satisfaction Scale (GUESS). Human Factors 58, 8 (2016), 1217-1247. https://doi.org/10.1177/0018720816669646 PMID: 27647156.

[6] Reddit. 2021. Reddit. Retrieved Jan 7, 2021 from https://www.reddit.com/

[7] Grand View Research. 2020. Virtual Reality Market Size, Share \& Trends Analysis Report By Device. Retrieved Jan 7, 2021 from https://www. grandviewresearch.com/industry-analysis/virtual-reality-vr-market

[8] William J. Shelstad, Dustin C. Smith, and Barbara S. Chaparro. 2017. Gaming on the Rift: How Virtual Reality Affects Game User Satisfaction. Proceedings of the Human Factors and Ergonomics Society Annual Meeting 61, 1 (2017), 2072-2076. https://doi.org/10.1177/1541931213602001

[9] Penny Sweetser and Zane Rogalewicz. 2020. Affording Enjoyment in VR Games: Possibilities, Pitfalls, and Perfection. In Proceedings of the 32nd Australian Conference on Human-Computer-Interaction (Sydney, NSW, Australia) (OZCHI'20). ACM, New York, NY, USA.

[10] Penelope Sweetser and Peta Wyeth. 2005. GameFlow: A Model for Evaluating Player Enjoyment in Games. Comput. Entertain. 3 , 3 (July 2005), 3. https://doi.org/10.1145/1077246.1077253

[11] Tom Wijman. 2020. The World's 2.7 Billion Gamers Will Spend \$159.3 Billion on Games in 2020; The Market Will Surpass \$200 Billion by 2023. Retrieved Jan 7, 2021 from https://newzoo.com/insights/articles/newzoo-games-market-numbers-revenues-and-audience-2020-2023/ 